\title{
Differences in the genetic control of early egg development and reproduction between C. elegans and its parthenogenetic relative $D$. coronatus
}

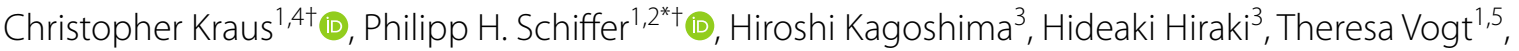 \\ Michael Kroiher ${ }^{1} \mathbb{B}$, Yuji Kohara ${ }^{3}$ and Einhard Schierenberg ${ }^{1}$ (i)
}

\begin{abstract}
Background: The free-living nematode Diploscapter coronatus is the closest known relative of Caenorhabditis elegans with parthenogenetic reproduction. It shows several developmental idiosyncracies, for example concerning the mode of reproduction, embryonic axis formation and early cleavage pattern (Lahl et al. in Int J Dev Biol 50:393-397, 2006). Our recent genome analysis (Hiraki et al. in BMC Genomics 18:478, 2017) provides a solid foundation to better understand the molecular basis of developmental idiosyncrasies in this species in an evolutionary context by comparison with selected other nematodes. Our genomic data also yielded indications for the view that $D$. coronatus is a product of interspecies hybridization.
\end{abstract}

Results: In a genomic comparison between D. coronatus, C. elegans, other representatives of the genus Caenorhabditis and the more distantly related Pristionchus pacificus and Panagrellus redivivus, certain genes required for central developmental processes in C. elegans like control of meiosis and establishment of embryonic polarity were found to be restricted to the genus Caenorhabditis. The mRNA content of early D. coronatus embryos was sequenced and compared with similar stages in C. elegans and Ascaris suum. We identified 350 gene families transcribed in the early embryo of $D$. coronatus but not in the other two nematodes. Looking at individual genes transcribed early in $D$. coronatus but not in C. elegans and A. suum, we found that orthologs of most of these are present in the genomes of the latter species as well, suggesting heterochronic shifts with respect to expression behavior. Considerable genomic heterozygosity and allelic divergence lend further support to the view that $D$. coronatus may be the result of an interspecies hybridization. Expression analysis of early acting single-copy genes yields no indication for silencing of one parental genome.

Conclusions: Our comparative cellular and molecular studies support the view that the genus Caenorhabditis differs considerably from the other studied nematodes in its control of development and reproduction. The easy-to-culture parthenogenetic D. coronatus, with its high-quality draft genome and only a single chromosome when haploid, offers many new starting points on the cellular, molecular and genomic level to explore alternative routes of nematode development and reproduction.

Keywords: Nematode, Caenorhabditis, Parthenogenesis, Embryogenesis, Polarity, Chromosome, Genome, Transcriptome, Hybridization

\footnotetext{
*Correspondence: philipp.schiffer@gmail.com; p.schiffer@ucl.ac.uk

${ }^{+}$Christopher Kraus and Philipp H. Schiffer equally contributing first authors

${ }^{2}$ Genetics, Evolution and Environment, University College London, London WC16BT, UK

Full list of author information is available at the end of the article
} 


\section{Background}

Development of the model Caenorhabditis elegans has been extensively studied. Although comparative studies in other nematodes revealed considerable variations on the cellular level (for review, see [3]), it seemed selfevident that gene cascades controlling development are conserved across the phylum. However, analysis on the levels of genome and transcriptome suggested major changes in the logic of cell specification and the action of Developmental System Drift [4], i.e., the modification of developmental processes due to altered gene regulatory networks without changing the phenotype of the emerging organism, even between nematodes from neighboring clades $[5,6]$. While $C$. elegans can obviously not serve as a general model for nematode development, it has remained unclear how fast the genetic control of development has changed during evolution in the longbranched roundworms.

Therefore, we here analyze molecular and cellular aspects of early development and reproduction in the parthenogenetic species Diploscapter coronatus, which has just half the body size of $C$. elegans and whose genome we described recently [2]. D. coronatus is a member of the Protorhabditis group, which not only belongs to the same clade as the genus Caenorhabditis but is the immediate sister taxon of it [7]. We previously described some idiosyncrasies in early development of $D$. coronatus using microscopic approaches $[1,8]$.

In the androdioecious hermaphrodite, C. elegans oocytes arrest in meiotic prophase and are released sequentially, this way delivering a continuous supply of maturing oocytes $[9,10]$. The generation of somatic founder cells via asymmetric germline divisions in $D$. coronatus takes place in the same way as in C. elegans despite the absence of sperm-induced polarization prior to first cleavage. In $D$. coronatus, only one polar body is generated during a truncated meiosis explaining the diploid status without fertilization. This suggests differences in the molecular machinery initiating axis polarity.

The control of oocyte maturation in C. elegans requires signaling from the sperm via major sperm protein (MSP) [11]. We found earlier that MSP genes are present in parthenogenetic nematodes, including $D$. coronatus; however, MSP protein could not be detected there [12].

Screening the gene and protein sets of $D$. coronatus for regulators of important developmental processes in $C$. elegans, we make comparisons with other members of the genus Caenorhabditis, as well as two more distantly related nematodes with gonochoristic and hermaphroditic reproduction. Particularly, we search for peculiarities that can be related to the development of oocyte and early embryo in the context of parthenogenetic reproduction in D. coronatus.
In a second approach, we compare the transcriptome of early embryonic stages in $D$. coronatus with the known complement of genes expressed in corresponding stages of C. elegans [13] and Ascaris [14]. In particular, we are interested to explore to what extent the expression of certain genes in $D$. coronatus can be correlated with its early developmental idiosyncrasies.

Nematodes can follow different modes of reproduction including parthenogenesis. This reproductive mode is a deviation of an original bisexual situation and has been established several times independently within different metazoan taxa. It can arise in several ways including spontaneous mutation, interspecies hybridization or infection with microorganisms and may go along with regular meiosis followed by fusion of gametes, or complete or partial suppression of meiosis [15]. However, no parthenogens have been found in the genus Caenorhabditis, despite the rapidly rising number of described species (> 30), while at the same time a shift from gonochoristic to hermaphroditic reproduction took place several times independently in this taxon $[16,17]$.

With our earlier finding in mind that the genome of $D$. coronatus shows a high degree of heterozygosity [2], we looked for further evidence that parthenogenesis in this species may be the result of interspecies hybridization which is considered a major route to this mode of reproduction in invertebrates and possibly the only one in vertebrates [18].

\section{Methods}

\section{Nematode culture and strains}

Strains were cultured on agar plates with the uracilrequiring OP50 strain of E. coli as a food source, essentially as described by Brenner [19], except that, to reduce contamination with other bacteria, we used minimal medium plates [8]. D. coronatus (PDL0010) was kindly provided by Paul De Ley, Dept. Nematology, University of California, Riverside.

To measure brood size, 17 juveniles of $D$. coronatus were isolated and grown individually as described above. When starting to lay eggs, animals were transferred to new culture plates every two days until they died and the total of hatched larvae was counted.

\section{Microscopical analysis and 3-D reconstructions}

Development was studied with Nomarski optics using a $100 \times$ objective. One-cell stage embryos were collected from agar plates with a drawn-out Pasteur pipette or after dissection of gravid adults. Specimens were placed on microscope slides carrying a thin agar layer as a mechanical buffer and covered with a coverslip sealed on the edges with petroleum jelly. Development was recorded using a 4D microscope with 15-25 optical sections/ 
embryo and 15-60 s time intervals between scans [20]. Cell behavior was traced with help of the Simi Biocell software (Simi Reality Motion Systems GmbH, Unterschleißheim, Germany). Nuclei were counted in optical sections of DAPI-stained isolated gonads.

\section{Diploscapter coronatus ITS, SSU, LSU rDNA analysis}

For each $D$. coronatus rDNA gene, two individuals were picked and lysed. Using single-worm PCR [21], we cloned sequences from each rDNA gene and individual into separate pBluescript KS cloning vectors. For amplification of the ribosomal small subunit (SSU), we used primers described in $[22,23]$, for the ribosomal large subunit (LSU) primers from [24] and for the ribosomal internal transcribed spacer (ITS) from [25]. For phylogenetic analysis, we used Mr. Bayes [26](version 3.1.2) and RAxML (version 7.2.8) [27] with standard parameters and 100 bootstraps. Resulting trees were collapsed after first node.

\section{OrthoMCL clustering and identification of the presence and absence of orthologs}

To reliably compare orthologs, we used the OrthoMCL pipeline (version 2.0.9) [28] to cluster proteomes of five Caenorhabditis species (C. angaria, C. briggsae, C. elegans, C. japonica, C. tropicalis [29-31]; http://www.ebi. ac.uk/ena/data/view/GCA_000186765.1), D. coronatus, Pristionchus pacificus [32], Panagrellus redivivus [33] and Ascaris suum [14,34]. The absence of genes in the $D$. coronatus genome which are present in the genomes of C. elegans, C. briggsae and C. remanei was confirmed by reciprocal BLAST search.

\section{Gene ontology (GO) term analysis}

Fisher's exact test for gene ontology (GO) terms of $D$. coronatus-specific clusters and singletons (proteins comprising a species-specific single variant) during early embryogenesis was applied to identify significantly overrepresented GO terms $[35,36](\mathrm{FDR}<0.05 ; p<0.001)$.

\section{Phylogenetic classification and analysis}

We here refer to the phylogeny of Holterman [37], dividing nematodes into 12 different clades. Following De Ley and Blaxter [38], we distinguish more basal Enoplea (clades 1 and 2) from more derived Chromadorea (clades 3-12). While C. elegans, D. coronatus and P. pacificus are members of clade 9 , other species mentioned in this paper belong to clade 12 (Meloidogyne spp.), clade 11 (Acrobeloides nanus), clade 10 (P. redivivus; Panagrolaimus spp.), clade 8 (A. suum), clade 6 (Plectus sambesii) and clade 2 (Romanomermis culicivorax).

In order to visualize the structural differences between the C. elegans and the D. coronatus LET-99 homologs, multiple alignments were performed using the program Clustal OMEGA [39]. Outgroup proteins including an $\mathrm{N}$-terminal DEP domain were retrieved from Pfam database [40]. For the phylogenetic analysis, the best amino acid (AA) substitution matrices were identified using the program Prottest3 under the conditions of invariant sites and gamma optimization. Best substitution matrices were identified under the condition of the Bayesian information criterion and the Akaike information criterion [41]. Phylogenetic trees were constructed using RAxML with gamma value optimization and the substitution matrices suggested by Prottest3. Each tree was bootstrapped 100 times.

\section{RNA extraction and RNA sequencing of selected embryonic stages}

For RNA sequencing, we collected under the dissecting scope four batches $(=4$ independent biological replicates) of approximately 100 eggs each, consisting of 1-8 cell stage embryos. These were placed into $25 \mu \mathrm{l} \mathrm{H}{ }_{2} \mathrm{O}$, shock-frozen in liquid nitrogen and immediately stored at $-80{ }^{\circ} \mathrm{C}$ to avoid RNA degeneration. RNA of each sample was extracted by a slightly modified version of an established protocol [42]. Instead of using $4 \mathrm{M}$ guadiniumthiocyanate (GU) buffer, we used $6 \mathrm{M}$ GU buffer. By adding $175 \mu \mathrm{l} 6 \mathrm{M}$ GU buffer and using a homogenizer (Ultra-Turrax, IKA Werke GmbH), it was possible to lyse the samples under chaotropic conditions. The extracted amount of total RNA was dissolved in $2 \mu$ l RNAse-free water and used for RNA amplification using the "Message AMP II" kit (AM1751; Life Technologies Inc.) following the protocol of Hashimshony et al. [13]. This allowed linear amplification (in contrast to exponential amplification methods such as PCR) of the total RNA content, hence significantly decreasing the amplification bias. TruSeq library construction (TruSeq preparation kit version 2; Illumina Inc.) and RNA sequencing were performed on Illumina HiSeq and MiSeq platforms at the local sequencing facility (CCG Cologne). Retrieved paired-end reads ranged from approximately 8,500,000 to $31,000,000$, depending on the sequencing platform.

\section{Post-sequencing analysis}

Illumina paired-end reads were retrieved in four independent sequencing assays. Illumina adapters and indexes were removed using the program Trimmomatic [43], and $5^{\prime}$ and $3^{\prime}$-prime error-prone reads were removed using the program sickle (github.com/najoshi/sickle). Trimmed reads were used to generate a transcriptome using the de novo assembler Trinity [44]. To identify even scarce transcripts all four libraries were combined, this way a transcriptome with a maximum number of transcripts and the highest median was obtained. To screen 
for and eliminate bacterial contamination, assembled transcripts were mapped back to the $D$. coronatus EST library and transcriptome using bowtie2 [45]. For comparison with similar early embryonic stages of $C$. elegans and A. suum, the raw data were taken from $[13,46]$. In the case of $C$. elegans, sequences showing an average TPM (transcripts per millions) value of $>5$ were counted as being expressed. In $A$. suum, significant expression differences between the 1- to 4-cell stages on the one hand and subsequent stages on the other [46] allowed an estimation of the early-stage-specific transcriptome.

The transcriptome of $D$. coronatus was translated into protein sequences using the program Transdecoder [44] with a minimum AA length of 49 residues. Transcripts for which information was only available for the $3^{\prime}$-UTR (untranslated region) or which were shorter than 49 residues were aligned to an EST library to generate extended gene models. Resulting extended contigs were translated into AA sequences following the same procedure as described above. For C. elegans and A. suum, proteins corresponding to the early transcriptome were downloaded from wormbase.org. The retrieved protein sequences were used for orthologous clustering using OrthoMCL.

\section{Identification of "allelic" gene variants}

We determined the intron-exon structure and the positions on the genome for all of the predicted genes. By aligning and clustering all EST libraries making use of CD-HIT [47] at a threshold of $90 \%$ identity, we identified ESTs belonging to the same gene. We mapped clustered ESTs against the genome using BLAT [48] this way confirming the exact position of each EST cluster on the genome. EST clusters mapping to open reading frames (ORFs) of genes were translated into AA sequences. Corresponding proteins were cross-compared by an all-versus-all blast [49] approach (at a threshold of 98\% identity). We sampled pairwise occurring genes with an amino acid identity of $>98 \%$. These were considered as different copies of the same gene under the condition that they were positioned on different contigs. In the following, we call these pairwise occurring genes with high AA identity "alleles." Taking into account the relative position on the genome, we deduced their numbers by counting positions and contigs.

\section{Identification of $C$. elegans genes in $D$. coronatus with two "alleles" at different loci}

Orthologous clusters consisting of only two proteins were extracted from our OrthoMCL clustering. Respective protein sequences were pairwise aligned via clustal OMEGA multiple alignment algorithms. AA identity was calculated by custom Perl scripts. Sequences which are known to exist at two different loci in the C. elegans genome and still have an identity of $95 \%$ were considered to be genes existing in two distinct "alleles."

\section{Single-copy gene analysis}

Single-copy genes present in nematodes of various clades as well as in Drosophila melanogaster and Saccharomyces cerevisiae were selected based on [50]. D. coronatus and $C$. remanei orthologs were identified by OrthoMCL. For C. remanei, candidates were retrieved from wormbase.org using the C. elegans orthologs described in [50]. Known $C$. elegans orthologs were identified in the $D$. coronatus genome with NCBI Blast. The identity of a $D$. coronatus ortholog was confirmed by OrthoMCL clustering and by using the predicted genes of $D$. coronatus as a query to search for the original ortholog in the C. elegans genome (best-reciprocal-blast-hit approach).

By pairwise alignment with clustalW [51], we compared on the one hand $D$. coronatus "alleles" with each other and on the other hand the respective C. elegans orthologs with their C. remanei orthologs. We also scanned the orthologs of the single-copy genes for conserved protein domains with InterProScan [52]. We counted the number of synonymous and non-synonymous mutations within the respective single-copy genes by pairwise alignment of the sequences on the nucleotide level taking into account the appropriate reading frame and by using the KaKs Calculator (version 1.2) with standard parameters (http://evolution.genomics.org.cn/ software.htm; [53]). For a statistics of average nucleotide exchange rates, the one-tailed Welch $t$ test for non-equal variances was applied. We tested specifically the null hypothesis, i.e., whether the fraction of non-synonymous mutations is equal or greater than the fraction of synonymous mutations. The null hypothesis was rejected at a significance level of $\alpha=0.01$.

\section{Identification of expressed $D$. coronatus "alleles" during early embryogenesis}

Diploscapter coronatus transcripts expressed during early embryogenesis were mapped back to the $D$. coronatus expressed sequence tag (EST) library using the program Bowtie2. Transcripts mapping to two different genomic contigs were considered as "alleles" (see above). ESTs were clustered using the program CD-HIT with standard parameters for $>94 \%$ identity. Mapped reads were used to identify polymorphisms (in particular singlenucleotide polymorphisms; SNPs) by using the programs SAMtools and Bcftools [54] with a minimum sequencing coverage of tenfold per site. The genotype quality (GQ, [55]) of each retrieved SNP was represented by a maximum likelihood for wrong SNP calls of $<10^{-3}$. Variations which did not meet these criteria were considered as 
random variants probably due to sequencing errors. Halplotypes for each variant were inferred by usage of the "vcfgeno2halpo" command of the Vcflib suite (https:// github.com/vcflib/vcflib) for a window size of $500 \mathrm{bp}$. Transcripts with $>99 \%$ nucleotide identity were defined as indistinguishable and excluded from the analysis.

\section{Results}

\section{Multi-species orthologous clustering}

We compared our $D$. coronatus data with other nematodes, including $C$. elegans, to better understand the molecular basis of developmental peculiarities in this species (Table 1 ). The D. coronatus draft genome contains more than 34,000 protein predictions, and we used these to screen for conserved and species-specific genes. In order to identify robust orthologous clusters in comparison with several other species selected for their phylogenetic position (Table 1, Fig. 1), we used OrthoMCL. In total, we found over 8000 orthologous clusters shared between $P$. redivivus (clade 10) and A. suum (clade 8). About $80 \%$ of these are present in all seven clade- 9 species considered here as well, suggesting a core set of shared protein families. However, the majority of the nearly 20,000 clusters are not shared with $P$. redivivus and A. suum (Fig. 2).

By analyzing five Caenorhabditis species and in addition D. coronatus, P. pacificus and P. redivivus, we found that over 5000 orthologous clusters, or nearly $50 \%$ of all clade 9-restricted clusters were specific to the genus $\mathrm{Cae}$ norhabditis. This suggests that during evolution a considerable number of genes must have newly arisen in the lineage leading to this taxon.

\section{Absence of genes and development in $D$. coronatus}

Using our ortholog clustering (Fig. 2), we investigated which genes known to be crucial in development of $C$. elegans are restricted to the genus Caenorhabditis. We found an absence of developmental regulators for a variety of biological processes in other nematodes (Fig. 3) and decided to focus on oogenesis and early embryogenesis where we had observed phenotypical idiosyncrasies in $D$. coronatus.

\section{Oocyte development and modified meiosis}

In C. elegans, the development of germ cells from mitotic oogonia to mature oocytes is arrested in prophase of meiosis I and their subsequent activation by sperm is an elaborate process [56]. We found that each of the two gonadal arms of the mature $D$. coronatus adult is about $5 \times$ smaller than in C. elegans and contains only 30-100 germ cell nuclei (Fig. 4a, b; $n=20$ ) in contrast to the latter where about one thousand is generated [57]. Under our laboratory conditions, individual Diploscapter
Table 1 Species and proteomes used in this study

\begin{tabular}{lcll}
\hline Species & Clade $^{\mathbf{a}}$ & \multicolumn{2}{l}{ Number of proteins } \\
\cline { 3 - 4 } & & Whole genome & Early embryogenesis \\
\hline C. elegans & 9 & 22,855 & 8817 \\
C. angaria & 9 & 28,371 & - \\
C. japonica & 9 & 30,083 & - \\
C. tropicalis & 9 & 24,532 & - \\
D. coronatus & 9 & 31,693 & 4610 \\
P. pacificus & 9 & 23,750 & - \\
A. suum & 8 & 17,555 & 3093 \\
P. redivivus & 10 & 26,372 & -
\end{tabular}

a For phylogenetic classification, see "Methods"

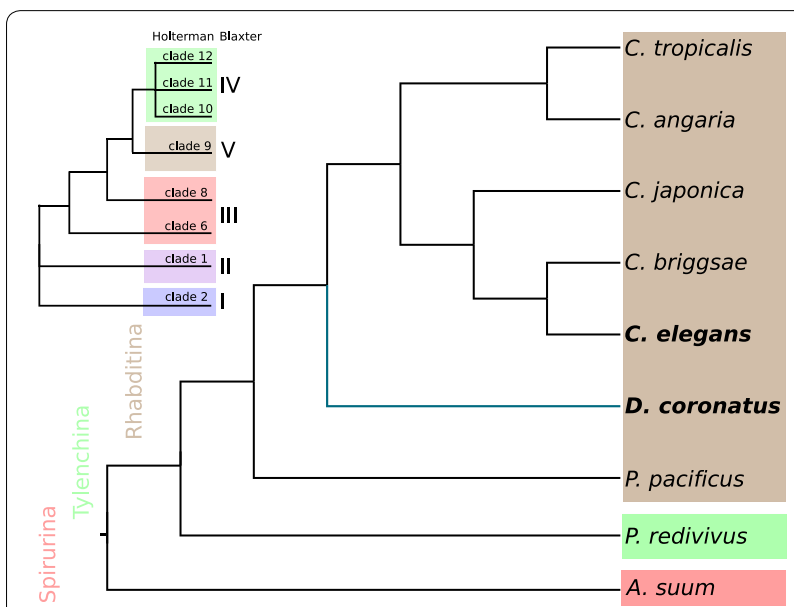

Fig. 1 Cladogram depicting all nematode clades relevant for this work after the Holterman [37] nomenclature (De Ley and Blaxter [38] nomenclature for comparison) and showing the phylogenetic relationships of species compared in our orthology analysis

females produced less than one-third the number of eggs found in C. elegans (on average 80; $n=17$ ). Different to the latter, the size of developing germ cells in $D$. coronatus increases only marginally except for the most mature one ("-1 oocyte") which is much larger and densely filled with yolk granules (Fig. 4a; in older adults also the -2 oocyte starts to grow). We wondered whether the same phases of oocyte differentiation as in C. elegans can be found in D. coronatus.

The analysis of DAPI-stained germline nuclei indicates that this is not the case. In the adult $D$. coronatus ovary essentially all germ cell nuclei appear to be in premeiotic interphase (Fig. 4b) except for rarely observed mitoses in the distal-most region and possibly initiation of meiosis in some late oocytes. Condensed meiotic chromosomes were only found in oval-shaped 1-cell stages in the uterus 


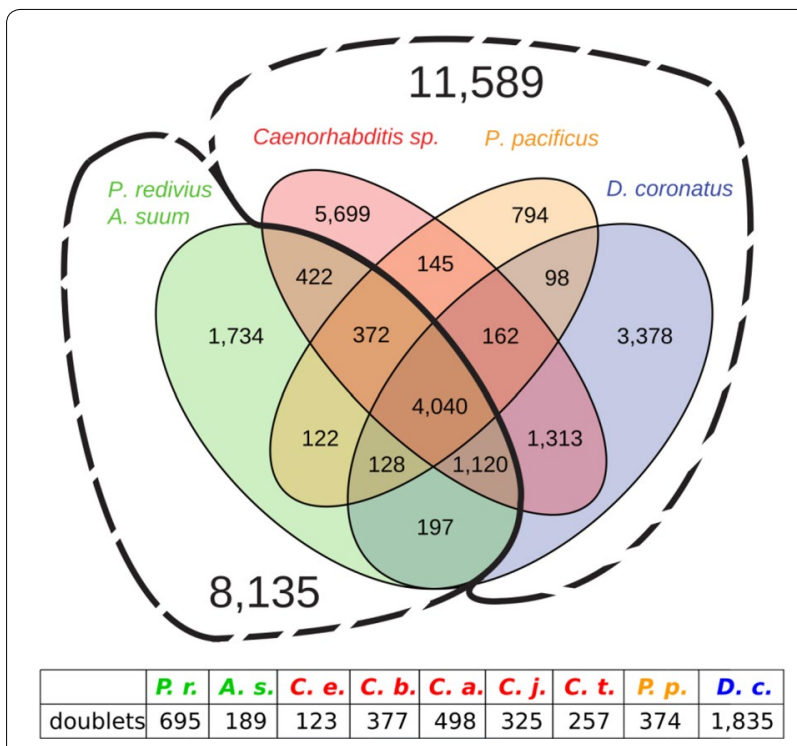

Fig. 2 Distribution of shared and specific orthologous clusters for representatives of Diploscapter coronatus (blue), Pristionchus pacificus (orange) and the genus Caenorhabditis (red), as well as for the outgroups Panagrellus redivivus (clade 10) and Ascaris suum (clade 8; both in green). Insert: Number of species-specific proteins present in two distinct "alleles" ("doublets")

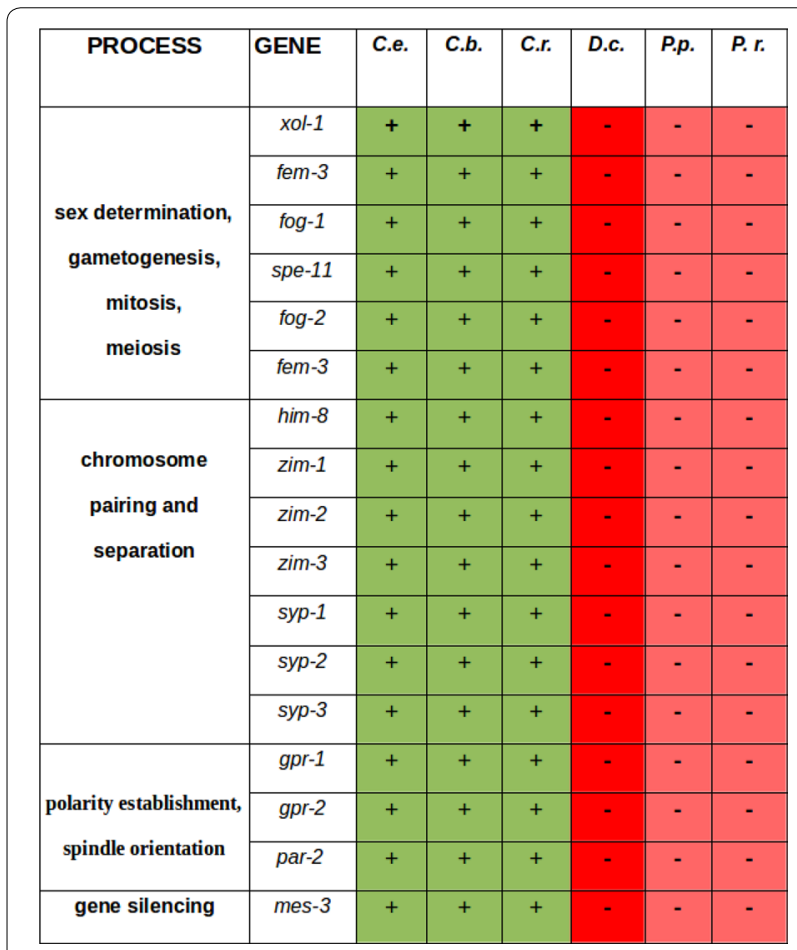

Fig. 3 Orthologs of genes with essential functions in different processes in C. elegans not found in D. coronatus and two other species outside the genus Caenorhabditis. Green, genes found; red, genes not found. C.e., C. elegans; C.b., C. briggsae; C.r., C. remanei; D.c., D. coronatus; P.p., P. pacificus; P.r., P. redivivus surrounded by an eggshell (Fig. 4c, d). Thus, in contrast to C. elegans, in D. coronatus individual germ cells seem to enter meiosis late and one by one without prophase arrest.

Diploscapter coronatus contains only two chromosomes in the diploid status $(2 n=2[2,58])$, whereas in C. elegans $2 n$ equals 12 chromosomes [59]. In accordance with Hechlers report [58], we never observed paired meiotic chromosomes.

Screening the $D$. coronatus genome for genes essential for germ cell development or sex-specific cell differentiation in C. elegans, we found orthologs of several of such genes missing (see detailed description below). However, their absence cannot serve as a straight forward explanation for special features of the parthenogenetic $D$. coronatus as they were not detected in Pristionchus and Panagrellus as well (for phylogeny, see Methods), while present in all three considered Caenorhabditis species (Fig. 3). At least, our data indicate that the control of central developmental processes differs between members of the genus Caenorhabditis and representatives of neighboring clades and even within the same clade.

\section{Polarity establishment and early embryogenesis}

Gonochoristic and hermaphroditic reproduction depends on sperm, which contributes the centriole, essential for mitotic spindle formation, and initiates embryonic polarity as a prerequisite for subsequent asymmetric cell division and soma/germline separation in C. elegans $[60,61]$.

The proper positioning of the first cleavage spindle in C. elegans and subsequent movement of one aster toward the posterior pole of the zygote preceding its asymmetric division requires the presence of LET-99. The C-terminal domain of LET-99 appears to be important for its functionality as nonsense mutations lead to strong phenotypes [62]. LET-99 is also the main regulator for the localization of LIN-5 and GPR-1/GPR-2 [63] which act together to generate the necessary spindle pulling force $[64,65]$. In the genome of $D$. coronatus, we could not find orthologs of gpr-1/2 (Fig. 3).

We identified a LET-99 homolog in D. coronatus (DcLET-99). However, alignment of the protein sequence with that of other species (Fig. 5) revealed that the last $70 \mathrm{AA}$ of the C-terminal region is absent in the genus Caenorhabditis. The Diploscapter ortholog thus has more similarity with the protein in other nematodes, like the ones included in our study (Fig. 5). This finding may reflect a non-equivalent function of this protein in C. elegans and D. coronatus.

With these findings in mind, we compared the establishment of asymmetry between $D$. coronatus and $C$. elegans. Characteristic for C. elegans is the migration 

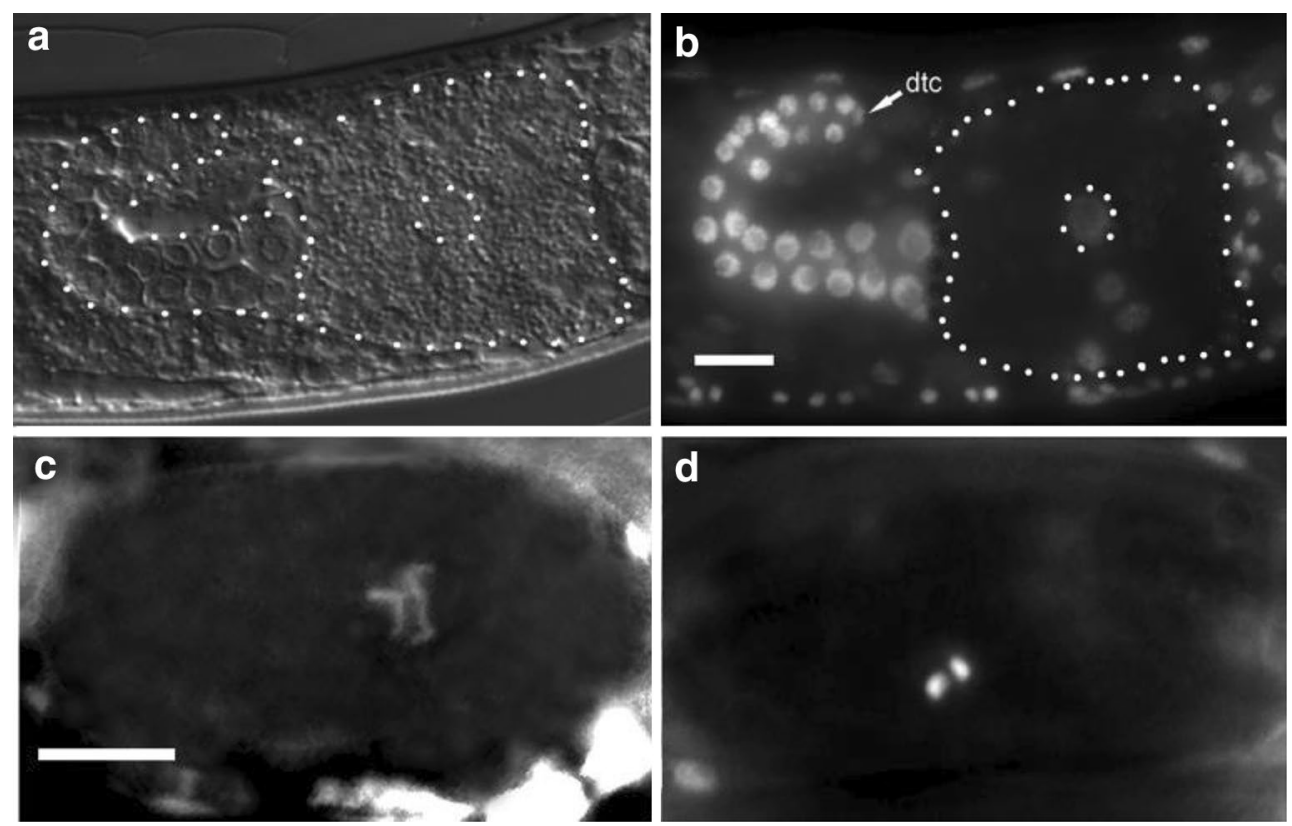

Fig. 4 Gonad and chromosomes in D. coronatus. a Outline of one gonadal arm including one large oocyte; b gonadal arm with DAPI-marked germ cell nuclei; c, $\mathbf{d}$ extended and condensed chromosomes in prophase of meiosis I

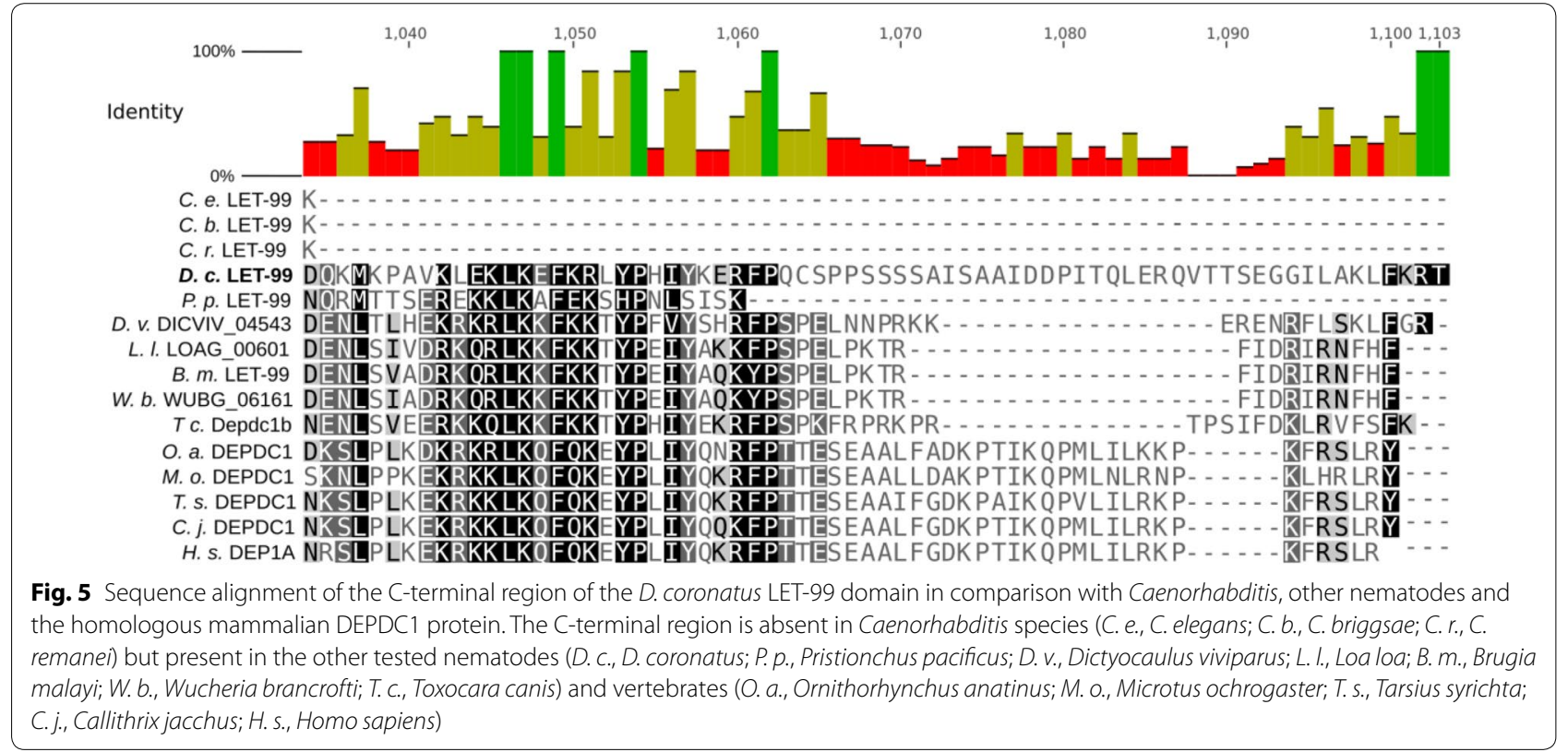

of the two pronuclei to the center of the fertilized egg cell and their subsequent fusion (Fig. 6a-c). Consequently, the posterior aster together with the future $\mathrm{P}_{1}$ chromosome set is quickly translocated to the posterior, while the anterior aster remains at its original position (Fig. 6c, d). This results in an asymmetric division of the zygote into a larger somatic $\mathrm{AB}$ and a smaller $\mathrm{P}_{1}$ germline cell (Fig. 5e, f). Subsequently, AB divides with transverse and $P_{1}$ with longitudinal spindle orientation (Fig. 6g) resulting in a rhomboid 4-cell stage (Fig. 6h). With the division of $\mathrm{P}_{2}$, a reversal of cleavage polarity (PR) takes place in the germline such that $\mathrm{P}_{3}$ occupies a more anterior position relative to its somatic sister $\mathrm{C}$ (Fig. 6i) [66]. 


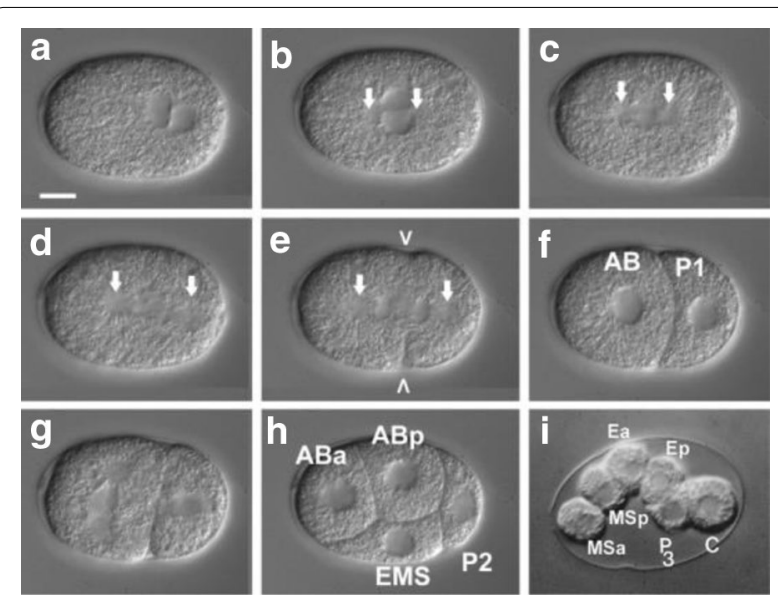

Fig. 6 Early embryogenesis of C. elegans. a pronuclei meet posteriorly; b central rotation of pronuclei; c central cleavage spindle; d posterior movement of posterior aster; e formation of two nuclei, initiation of cytokinesis; $\mathbf{f}$ 2-cell stage; $\mathbf{g}$ spindle orientation: transverse in $\mathrm{AB}$ and longitudinal in $\mathrm{P} 1 ; \mathbf{h}$ 4-cell stage; i polarity reversal with generation of anterior $\mathrm{P} 3$ and posterior $\mathrm{C}$. For further details, see text. Arrows, centriolar regions; arrowheads, cleavage furrow; i for better visualization of polarity reversal in the germline $A B$ cells had been removed through a laser-induced hole in the eggshell. Scale bar, $10 \mu \mathrm{m}$; orientation, anterior, left
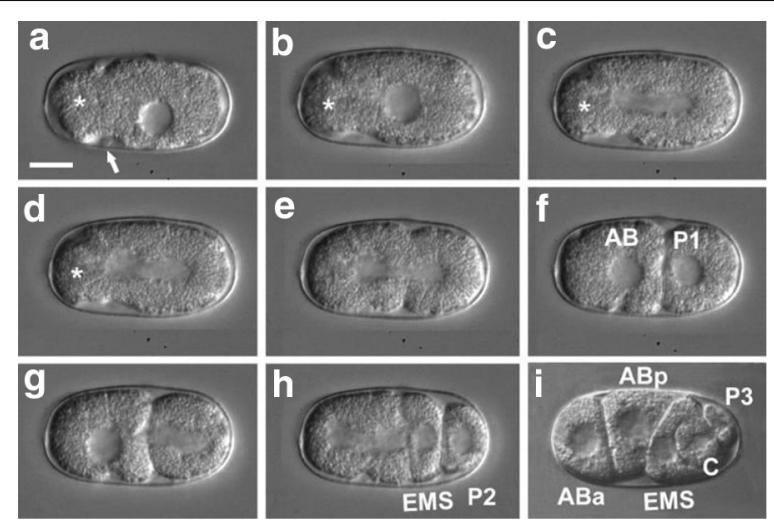

Fig. 7 Early embryogenesis of D. coronatus. a single pronucleus; b formation of anterior constriction; c cleavage with central spindle; d formation of 2 nuclei, regression of constriction; e cytokinesis; $\mathbf{f} 2$-cell stage; $\mathbf{g}, \mathbf{h}$ a-p-oriented cleavage spindle in $\mathrm{P} 1$ and $\mathrm{AB} ; \mathbf{i}$ no polarity reversal with generation of anterior $C$ and posterior $P$ 3. For further details, see text. Arrow, single polar body; asterisk, separated anterior cytoplasm. Scale bar, $10 \mu \mathrm{m}$. Orientation, anterior, left

In the parthenogenetic Diploscapter, only one pronucleus is generated during meiosis (Fig. 7a, b; [1]). In contrast to C. elegans, a temporary constriction forms at the anterior pole and the maternal pronucleus occupies a slightly eccentric position (Fig. 7b-d). The zygote divides with no shift of the posterior aster (Fig. 6c-e), while the constriction regresses. This way, a larger $A B$ and $a$ smaller $\mathrm{P}_{1}$ cell are formed (Fig. 7e, f). Subsequently, $\mathrm{AB}$ divides with longitudinal spindle orientation like $\mathrm{P}_{1}$ (Fig. 7g, h) and a PR in $\mathrm{P}_{2}$ is absent (Fig. 7i). The absence of gpr-1 and gpr-2 plus the considerably diverged let-99 could give an explanation for the different ways of how the asymmetric division of the zygote is achieved in the two species.

As gpr- 1 and $g p r-2$ are absent in P. pacificus and P. redivivus, too (Fig. 3), we studied formation of asymmetry in 1-cell embryos there and found it to be similar to $D$. coronatus, while C. briggsae and C. remanei behave like C. elegans. This can also be deduced from the video clips accompanying [85]. In addition, we analyzed one representative each of Cephalobidae (A. nanus) and Plectidae (P. sambesii). With respect to spindle movement, they behave similar to $D$. coronatus (data not shown). These findings indicate that the genus Caenorhabditis has developed a special way of how to accomplish the first asymmetric cleavage.

\section{Spindle orientation and germline polarity}

As the same a-p spindle orientation in the AB cell of $D$. coronatus was also found in a par-3 mutant of C. elegans [67], we screened the genome of $D$. coronatus for the presence of par genes. We found an ortholog of par3 but not of par-2. The absence of these two genes in $C$. elegans leads to a transverse spindle orientation in P1 [67]. However, in the par-2/let-99 double mutant the majority of embryos orients the cleavage spindle longitudinally in both blastomeres [68]. As the same genes missing in $D$. coronatus are also absent in Pristionchus and Panagrellus, which, however (Fig. 3), show a C. elegans-like AB spindle orientation, the identified molecular differences between C. elegans and D. coronatus can at most be considered a prerequisite for an alternative spindle orientation. The visible presence of a central cortical region in $\mathrm{P} 1$ and $\mathrm{AB}$ rather than in P1 alone has been suggested to be responsible for capturing spindle microtubules in both cells of $D$. coronatus resulting in a-p spindle orientation [8].

\section{Early transcriptome: species-specific orthologous clusters and their expression}

To investigate to what extent the initial steps of embryogenesis in $D$. coronatus are reflected on the gene expression level, we sequenced 1-8-cell stages and compared their transcriptome with available data of similar stages from C. elegans [13] and A. suum [46]. By assembling transcriptomes from four independent biological replicates (Additional file 1: Table S1), we retrieved in total more than 6500 transcripts with a median length of 381 bp (Additional file 2: Fig. S2; Table 2). For around 70\% of these, we could identify open reading frames (ORFs) allowing a successful inference of the early proteome. 
Table 2 Average transcript lengths and numbers of transcripts of four independently sequenced $D$. coronatus libraries for early developmental stages

\begin{tabular}{llll}
\hline Libraries & Median transcript length (bp) & Mean transcript length (bp) & Number of transcripts \\
\hline 1 & 230.5 & 244.3 & 1059 \\
2 & 269.0 & 285.9 & 3151 \\
3 & 330.0 & 360.2 & 4550 \\
$1+3$ & 329.0 & 359.1 & 5218 \\
4 & 350.0 & 370.8 & 5384 \\
$2+4$ & 358.0 & 377.0 & 5531 \\
$3+4$ & 378.0 & 432.5 & 6213 \\
$1+3+4$ & 378.0 & 400.4 & 6487 \\
$1+2+3+4$ & 381.0 & 404.0 & 6550 \\
\hline
\end{tabular}

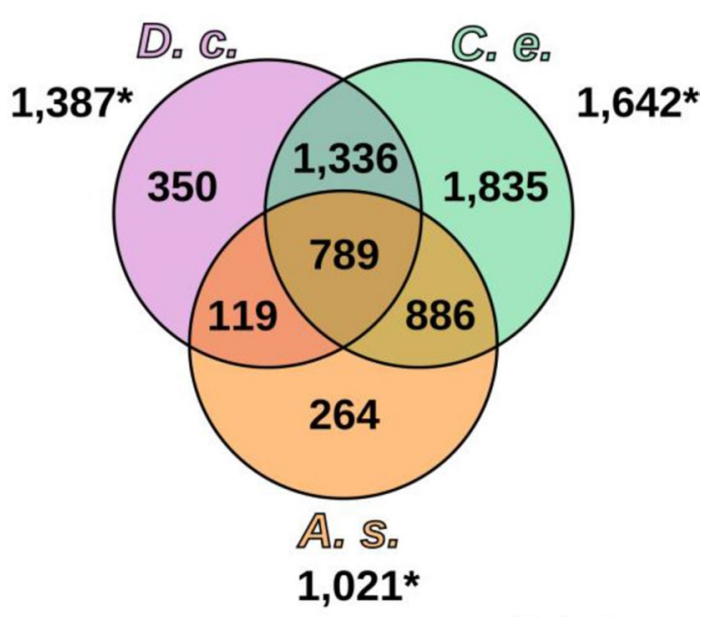

${ }^{*}$ ) singletons

Fig. 8 Early expressed clusters (protein families) of D. coronatus in comparison with the model C. elegans and the parasitic A. suum. For reference, the number of singletons is given for each species. C. e., Caenorhabditis elegans; D. c., Diploscapter coronatus; A. s., Ascaris suum

We used our transcriptome data from $D$. coronatus to perform an orthology clustering between the three nematodes. This way, we identified protein clusters that are shared among all three species as well as ones that are exclusively expressed in individual representatives during early development (Fig. 8).

By subtracting orthologs expressed during early embryogenesis in C. elegans, we identified genes expressed only in the other two species. We retrieved 119 orthologous clusters shared between $D$. coronatus and A. suum as well as 350 (comprising nearly 1500 genes) expressed exclusively in the early $D$. coronatus embryo (Fig. 8). While we found that orthologs of more than $60 \%$ of these genes are present in the genomes of C. elegans and A. suum, they are not transcribed there during early embryogenesis, suggesting interspecific heterochronic shifts of expression patterns.

Exploring which potential functions these early expressed genes might have in D. coronatus, more than 500 were classified as "unknown" as either no homology to any protein could be detected or homologous proteins are also unknown in their respective functions. In addition, we found over $1300 \mathrm{D}$. coronatus-specific transcripts expressed as a single sequence only ("singletons," Fig. 8).

\section{Early transcriptome: functional classification}

We searched for significantly overrepresented gene ontology (GO) terms for genes specifically expressed in the early $D$. coronatus embryo ( $1-8$ cell stage) in comparison with the full $D$. coronatus gene set (Additional file 3: Fig. S1, Additional file 4). By far, the most overrepresented was "regulation of centromere complex assembly" (GO:0090230; > 200-fold). Related to this is "CENPA containing nucleosome assembly at centromere" (GO:0034080; 48-fold). While it must be assumed that CENP-A is ubiquitously essential for mitosis, the lack of expression in C. elegans can be explained most easily with a maternal supply of the protein. Other potentially interesting overrepresented terms are "cytokinesis, initiation of separation" (GO:0007108; 64-fold) as well as chromatin remodeling-associated terms such as "histone $\mathrm{H} 2 \mathrm{~A}$ acetylation" (GO:0043968; 48-fold) and "NuA4 histone acetyltransferase complex" (GO:0035267; sixfold).

$\mathrm{NuA} 4$ is involved in the acetylation of $\mathrm{H} 2 \mathrm{~A}$ in yeast nucleosomes to exchange H2A for H2A.Z [69] which in turn regulates gene expression. In the $C$. elegans, embryo H2A.Z (or HTZ-1) is expressed in every blastomere and essential for normal development [70]. The observed NuA4-dependent acetylation in the early $D$. coronatus embryo is consistent with the assumption that massive zygotic transcription is required. 
In search for further genes that could play a role for the unique $D$. coronatus early development, we looked for genes that are expressed in the early embryo but for which no orthologs were found in the genomes of C. elegans and A. suum and analyzed their expression. In this category, we detected less than 10 genes. As we could not retrieve orthologs of any of these genes in other reference systems like Drosophila, zebrafish or mouse, we are presently unable to speculate about their function in Diploscapter.

As an alternative approach, we have started to look for protein domains significantly enriched in the early transcriptome of $D$. coronatus in comparison with C. elegans and A. suum. So far, we found a variety of enriched domains giving the chance to further investigate the role of certain proteins for developmental peculiarities in this species.

\section{The parthenogenetic $D$. coronatus shows high "allelic" divergence}

Analyzing the genome of $D$. coronatus, we had observed an unexpected degree of heterozygosity in the highquality draft genome (N50 =1,007,652 bp, number of scaffolds $=511 ;[2]$ ) resulting in the prediction of two "alleles" per gene in the Augustus pipeline. We observed a similar pattern when using Sanger sequencing methods on cloned PCR products of rRNA genes from individual

a

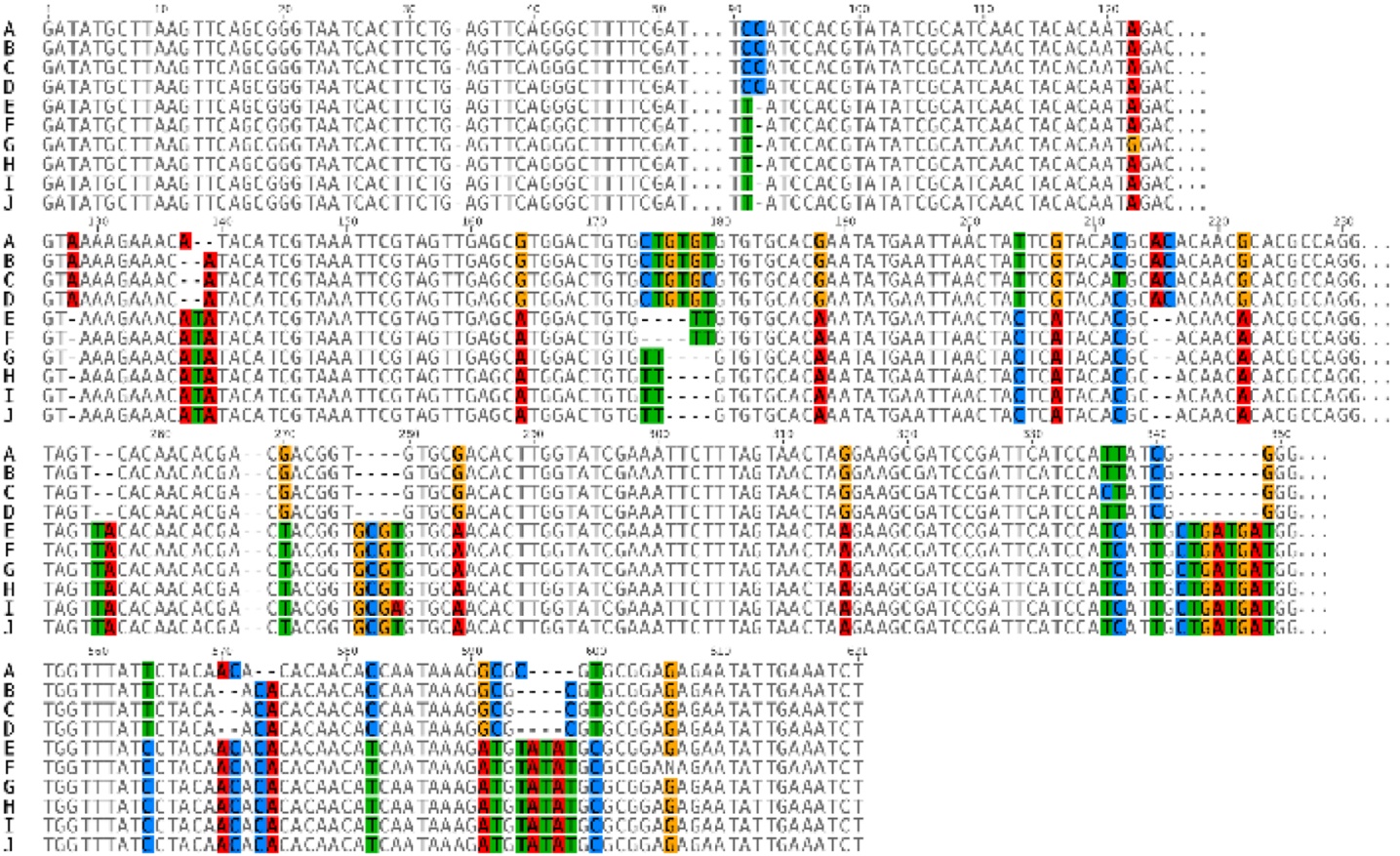

b

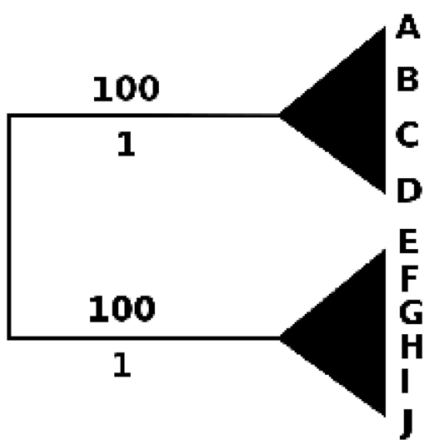

Fig. 9 Sequence comparison of the small internal transcribed spacer (ITS) rDNA gene of D. coronatus. a Sequence alignment of individual clones $(A-\nearrow$ ) shows selected regions with distinct single-nucleotide polymorphisms (SNPs). b Collapsed maximum likelihood tree representing clustering of sequenced clones. Bootstrap values are shown above and posterior probability beneath branches 
Table 3 Genes existing in two distinct alleles identified in the C. elegans genome

\begin{tabular}{lll}
\hline wormbase ID allele $\mathbf{1}$ & wormbase ID allele 2 & AA identity $^{\mathbf{a}}$ \\
\hline WBGene00004451 & WBGene00012179 & 97.82 \\
WBGene00001581 & WBGene00019017 & 96.01 \\
WBGene00019254 & WBGene00012795 & 96.20 \\
\hline
\end{tabular}

a At a threshold of at least $95 \%$ AA identity
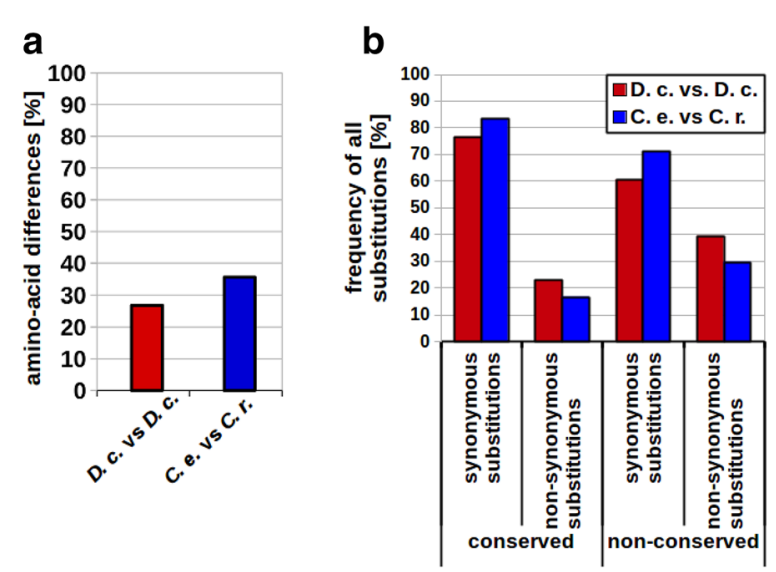

Fig. 10 Analysis of Diploscapter coronatus genes. a AA differences among alleles of single-copy genes of $C$. elegans versus $C$. remanei (blue; $n=229$ ) and the two "alleles" of D. coronatus orthologs (red; $n=307$ ); b percentage of synonymous and non-synonymous substitutions in non-conserved and conserved protein regions. C. e., $C$. elegans; C. r., C. remanei; D. C., D. coronatus

worms. In fact, we retrieved several sequences per rRNA locus per individual (Fig. 9a; Additional file 2: Fig. S2). Aligning these sequences showed distinct single-nucleotide polymorphisms (SNPs), and in a phylogenetic analysis all sequences could be allocated to one of two distinct "alleles" (Fig. 9b; Additional file 2: Fig. S2).

This was on a larger scale reflected in the orthology clustering we performed for this work. Here, we found more than 3000 D. coronatus-specific clusters and more than $50 \%$ of these contained two in-paralogs ("doublets"), which is a multiple of what has been observed in the other studied nematodes (e.g., 2\% in C. elegans; Fig. 2). In the complete genome, $66 \%$ of all genes in D. coronatus were found to exist in doublets [2]. In contrast, the number of clusters consisting of a species-specific single protein ("singletons") was by far the smallest in D. coronatus (2727; C. e. 6067).

Diploscapter coronatus-specific clusters comprising two proteins are in accordance with our earlier finding that in this species most genes are present as allelic pairs. In contrast, in C. elegans we found only three such examples (Table 3).
This pattern can be explained in two ways: (1) the independent accumulation of SNPs in non-recombining alleles (known as Meselson effect) in an old parthenogenetic lineage $[71,72]$ or (2) a hybrid origin of the parthenogenetic strain, where distinct alleles are inherited from the ancestral sexual species and genomic heterozygosity is maintained.

To investigate to what extent an accumulation of mutations occurred in the $D$. coronatus genome, we compared conserved single-copy genes in the nematode phylum. We found that in $D$. coronatus each of these single-copy genes exists in two distinct "alleles." The number of AA differences between the two $D$. coronatus "alleles" is similar to corresponding AA differences between C. elegans and C. remanei (Fig. 10a). Calculation of $\mathrm{dN} / \mathrm{dS}$ ratios in 11 arbitrarily selected single-copy genes revealed a median value of 0.158 (Additional file 1: Table S2) indicating negative selection. To identify potential differences between conserved and non-conserved protein domains, we applied InterProScan [52]. As expected, we found the number of non-synonymous exchanges to be lower than synonymous exchanges. But the ratio of synonymous to nonsynonymous substitutions was again not significantly different when comparing the two D. coronatus "alleles" with the respective $C$. elegans versus $C$. remanei orthologs (Fig. 10b). This is in line with (ii; see above) and can be easiest explained with a recent interspecies hybridization event.

\section{Differential expression of alleles}

Incipient hybrids may face dosage compensation issues, and proteins built from alleles inherited from different genomes might be incompatible or less efficient in molecular machineries. It is therefore possible that hybrid species need to silence one of the parental genomes [73]. Making use of the high-quality $D$. coronatus genome and our RNA-Seq data covering early embryogenesis we asked whether transcripts of one or both "alleles" are generated by screening for single-nucleotide polymorphisms (SNPs) in comparison with an EST library. We found that in the vast majority of genes, both "alleles" are expressed (Additional file 5: Fig. S3).

\section{Discussion}

Our previous studies of the parthenogenetic nematode D. coronatus focused on early embryogenesis $[1,8]$ and on molecular regulators important for the oocyte-toembryo transition [12]. Recently, we sequenced and assembled the genome of $D$. coronatus [2] and here use these data as a reference to revisit these questions in a more comprehensive scope. 


\section{Meiosis, $D$. coronatus-specific genes and preservation of heterozygosity}

Previously, it has been shown that $D$. coronatus passes through a truncated meiosis generating only one polar body [1]. We find that the gonad of $D$. coronatus differs in several aspects from $C$. elegans. The uniformity of germ cells (Fig. 4a, b) suggests that due to the small size of the gonad the distal tip cell (dtc) prevents entrance into meiosis essentially all along the gonadal tube (in contrast to C. elegans, see [74]). Only the most proximal oocyte, possibly due to its translocation into the uterus, has clearly visible escaped its influence (Fig. 4c, d). Another conclusion is that there is no meiotic arrest of germ cells in the gonad as found in C. elegans [56, 75], and therefore, no sperm is needed to lift it.

Compared to C. elegans, D. coronatus seems to follow a different strategy for the control of germ cell development. It would be attractive to study in this respect the role of the dtc in nematodes with particularly small gonads and a low brood size like $D$. coronatus or the parthenogenetic P. sambesii [76] and match it with representatives possessing extremely long gonadal arms, like Ascaris, producing millions of eggs [77].

The fact that no orthologs were found of crucial genes required for the generation of the synaptonemal complex (e.g., syp-1/-2/-3) and chromosome-specific adapters which are also involved in proper meiosis $($ zim-1/-2/-3, him-8; also see [2]) could mean that no crossing-over, and thus, no recombination takes place. This is consistent with our inability to detect paired meiotic chromosomes (see also [58]). However, the finding that the genes listed above are absent in Pristionchus and Panagrellus as well (Fig. 3) does not offer a straight forward explanation. The genetic control of meiosis (and other processes) seems to differ generally between Caenorhabditis, and other nematodes and may involve other genes.

We identified more than 3300 D. coronatus-specific clusters comprising more than 7500 genes in its genome. However, the role of most of these remains elusive since no orthologs have been found in other model systems (Fig. 2). Looking at early expressed genes alone, we recovered more than 500 genes of unknown function (Fig. 8).

A possible explanation for how the heterozygosity in D. coronatus can be preserved while passing through just one meiotic division, including the separation of chromatids rather than homologous chromosomes during meiosis I ("inverted meiosis"; [78]), has been discussed in [2].

\section{Polarity, asymmetry and absence of orthologs}

Microscopical analysis of early embryogenesis in D. coronatus revealed certain idiosyncrasies $[1,8]$. Here we show that the process of initial polarity establishment during the 1-cell stage differs markedly from C. elegans (Figs. 4,
5). Based on our ortholog clustering of the D. coronatus genome and eight other nematode genomes (Fig. 2), we conclude that certain genes crucial for early embryogenesis in C. elegans are absent in D. coronatus, P. pacificus, $P$. redivivus and $A$. suum (Fig. 3). With respect to polarity establishment, we did not find orthologs of essential genes known from C. elegans, such as $g p r-1 /-2$ in these species indicating differences in how early asymmetry is achieved. This appears particularly plausible for the parthenogenetic $D$. coronatus, where sperm as initial trigger is missing and where orientation of the anterior-posterior egg axis is obviously specified in a random fashion [1]. However, by scanning the $D$. coronatus genome for known GoLoco domain proteins involved in mitosis in vertebrates, we found that the human GPR-1/2 homolog LGN [60] has orthologs in D. coronatus (Additional file 6: Fig. S4). This suggests that C. elegans acquired new adaptor proteins for division, while $D$. coronatus relies on the established set of proteins known from outgroup species. It remains to be determined whether LGN functionally replaces GPR-1/2 in the $D$. coronatus 1 -cell stage and to what extent the modified dcLET-99 homolog (see "Results" and Fig. 5) is involved in the establishment of early polarity.

Looking at conserved protein complexes which are essential for maintaining already established polarity, such as PAR-3/PAR-6/PKC-3 or PAR-1/-2 [63], we found respective orthologs in $D$. coronatus, except for PAR-2 (Fig. 3). This result is in accordance with earlier studies, where it was proposed that the PAR-2/-3 system known from $C$. elegans evolved specifically in the genus Caenorhabditis [5]. What could be an alternative mechanism for establishing polarity in other nematodes like $D$. coronatus? It was shown earlier that in C. elegans the par-2 function can be replaced by $l g l-1$ (ortholog of the tumor suppressor gene lethal giant larvae) if it is overexpressed [79]. $l g l$ is known from various animal systems as a regulator of asymmetric cell division [80] indicating its high conservation. Its presence and the simultaneous absence of par-2 in D. coronatus suggest an ancestral molecular mechanism of asymmetric cleavage there and in the other non-Caenorhabditis species studied, as known from animals outside the nematodes $[81,82]$.

Another idiosyncracy in $D$. coronatus development is that the spindle in $A B$ performs the same rotation as in $\mathrm{P}_{1}$ resulting in its a-p orientation. In C. elegans, spindle microtubules in $\mathrm{P}_{1}$ seem to compete for attachment to a bleb-like site at the anterior cortex $[83,84]$. The presence of a prominent clear cortical region in the cortex of both 2-cell blastomeres in D. coronatus may indicate a symmetric distribution of components responsible for capturing spindle microtubules [8]. In the more basal nematode $R$. culicivorax (clade 2), a comparable effect 
on spindle behavior appears to be exerted in the 2-cell stage by the "region of the first midbody." Its ablation results in a perpendicular spindle orientation [85]. More data on the gene regulatory networks and intracellular constraints in $D$. coronatus should help us to find the molecular basis for the aberrant orientation of the cleavage spindle in $A B$. Presently, we speculate that here an original mechanism has been replaced in more derived nematodes, including C. elegans, while the phylogenetic branch comprising Diploscapter and Protorhabditis [7, $16,86]$ constitutes an atavism.

\section{GO term analysis}

We performed a gene ontology analysis of the "early transcriptome" and found over 70 terms overrepresented specifically in $D$. coronatus. It appears likely that enriched terms inform about important underlying biological processes [87]. While this approach should increase the likelihood for identifying such essential events, it can only play an advisory role in finding the most relevant, enriched annotation terms [88].

Many of the highly overrepresented terms are associated with chromosomal function in $D$. coronatus, for instance concerning centrosomes, chromosome structure, telomeres, DNA replication and gene regulation. This is consistent with the view that in $D$. coronatus these aspects differ considerably from C. elegans.

\section{Origin of parthenogenesis and reduction in chromosomes}

Different mechanisms and conditions have been described that could lead to the establishment of parthenogenesis $[15,89,90]$.

In the parthenogenetic $D$. coronatus where neither males nor sperm have been observed but a high degree of heterozygosity, we conceive two possible scenarios. One is hybridization of closely related gonochoristic species followed by the evolution of parthenogenesis and the other a spontaneous origin of parthenogenetic reproduction followed by independent accumulation of mutations in alleles. In the latter case, the $\mathrm{d} N / \mathrm{d} S$ ratio in the studied single-copy genes should be high as both non-synonymous and synonymous mutations are expected to accumulate to a similar degree in either of the two alleles. In contrast, a recent interspecies hybridization event should still show the footprints of purifying (negative) selection acting in the sexually reproducing parent species [91].

Comparing the AA sequences of "alleles" of arbitrarily selected single-copy genes, we found a high level of heterozygosity and the fraction of AA exchanges in these $D$. coronatus "alleles" was in fact not significantly different from that of orthologs in two distinct Caenorhabditis species (Fig. 10a).
This high genomic heterozygosity with two distinguishable "alleles" per gene, including the rDNA genes (Fig. 8; Additional file 2: Fig. S2) and single-copy genes (Fig. 10), and the low $\mathrm{dN} / \mathrm{dS}$ ratio, can be easiest explained with a recent event of interspecies hybridization between two closely related representatives where each parental genome has still preserved a major part of its ancestral functionality. In fact, a similar explanation has recently been suggested for more distantly related parasitic nematodes of the genus Meloidogyne [72, 92, 93]) and for more closely related parthenogens within the genus Panagrolaimus (bioRxiv: [94]).

$D$. coronatus possesses only a single chromosome in the haploid set, while C. elegans and most other studied free-living nematode species of clades 9-12 contain 6 chromosomes or more $[95,96]$ (our unpublished data). However, a close relative of Diploscapter, Protorhabditis sp. (laboratory strains JB 122), also contains just a single chromosome, while other members of this genus have six like C. elegans (E.S., unpublished data). The most parsimonious explanation for this minimal number in selected species is a comprehensive chromosome fusion. Very recently good evidence has been presented in another Diploscapter species that this may be in fact the case [97]. While a reduction in chromosome number due to fusion has been described in a variety of cases [98, 99], integration of all chromosomes into a single one would be a very extraordinary case deserving further attention. A combined detailed phylogenetic and chromosome analysis may reveal whether the assumed fusion has occurred once in a common ancestor or several times independently, whether this has been a stepwise modification or a single total fusion event, and how original chromosomes are arranged in this new construct. While we can only speculate about a possible mechanism for such a dramatic event, it may be worthwhile to investigate whether each chromosome in $D$. coronatus represents a complete haploid parental genome which has remained intact and functional due to the absence of recombination. In this case, chromosome fusion would have taken place most likely prior to the envisaged interspecies hybridization.

A haploid chromosome number of $n=1$ is neither a necessary prerequisite for parthenogenesis in nematodes nor a consequence of it, since in the bisexual Ascaris $(n=2)$ a variant exists with $n=1$ (var. univalens; [100]), and in the parthenogenetic species $A$. nanus and $P$. sambesii, we counted $n=6$.

It is an exciting question why in contrast to the closely related genera Diploscapter and Protorhabditis no parthenogenetic representatives have been found among the more than 30 Caenorhabditis species isolated so far [101] (NCBI Taxonomy). Taking into account the many 
idiosyncrasies of the taxon Caenorhabditis concerning the control of development (see, e.g., Fig. 3), it seems not unlikely that its molecular circuitry, allowing for instance a particularly rapid propagation, has not allowed the establishment of parthenogenesis.

\section{The genus Caenorhabditis versus Diploscapter and other nematodes}

Many C. elegans genes not found in D. coronatus were also absent from other non-Caenorhabditis species (Fig. 3). The peculiarities in early development of $D$. coronatus can thus not be explained just by the absence of these genes. It is feasible that in Diploscapter certain processes differ from C. elegans due to its parthenogenetic mode of reproduction. However, in light of the genomic similarities all studied non-Caenorhabditis nematodes may use the same alternative pathways (or at least alternative components) to control essentially identical developmental processes during oogenesis and early embryogenesis (Developmental System Drift [4]). Therefore, it appears more likely that these differences gave the freedom to establish parthenogenesis while preventing it in the genus Caenorhabditis. So far only part of the identified differences on the molecular level can be correlated with the described variations on the cellular level where "many roads lead to Rome" [102, 103]. Future studies have to reveal to what extent the developmental characteristics of $D$. coronatus can be explained with variations on the transcriptional and post-transcriptional level.

Even if with improved methods in the one or other case a credible ortholog of a C. elegans gene could be excavated that we here counted as being absent with our approach in the non-Caenorhabditis representatives, the differences on the level of genes and gene products in comparison with Diploscapter (and the other studied nematodes) will still remain remarkable. The particularly rapid evolutionary diversification in the genus Caenorhabditis [16] may be related to multifaceted opportunistic life styles. In permanently changing environments, this allows on the one hand short generation times and large progenies whenever food is available in abundance and on the other hand long-term survival under harsh conditions. Extended studies on additional Caenorhabditis species and close outgroups will have to reveal whether this genus is really as uniform with respect to its control of development as our limited studies suggest and at which branching points in the phylogenetic tree novelties arose. If the methodology to establish a transcriptional lineage of the early $C$. elegans embryo [104] is applicable to D. coronatus and other nematodes as well, evolutionary changes in time and space could even be traced on a single cell level.

\section{Conclusions}

The parthenogenetic $D$. coronatus reveals a variety of differences compared to C. elegans on the level of cells, chromosomes, genome and transcriptome indicating alternative routes for nematode development and reproduction. Thus, it appears to be an attractive study object to better understand the intricate pathways of evolutionary change among closely related species. Our comparative study further supports the view that the genus Caenorhabditis cannot be taken as a blueprint for the genetic control of developmental and reproductive processes in nematodes as it shows a number of idiosyncrasies absent in the other studied representatives. Future avenues to follow in order to reveal further developmentally relevant differences between $D$. coronatus, C. elegans and other rhabditid nematodes could be: (1) the role of early transcription versus maternal supply, (2) structure and function of the single chromosome $(n=1)$ in D. coronatus (Fig. 4c) assumed to be the result of fusion, (3) meiotic pairing and crossover, apparently absent in D. coronatus, (4) the mechanism of chromosome separation.

\section{Additional files}

Additional file 1: Table S1. RNA-Seq statistics. Table S2: $d N / d S$ values. Additional file 2: Fig. S2. a, C, Sequence comparison of the small (SSU) and large subunit (LSU) rDNA genes of D. coronatus. b, Collapsed Maximum Likelihood (ML) tree representing clustering of sequenced clones for the SSU rDNA gene. (d) Collapsed ML tree representing clustering of sequenced clones for the LSU rDNA gene. Bootstrap values are shown above and posterior probability values beneath branches.

Additional file 3: Fig. S1. GO terms enriched in the D. coronatus 1-8 cell transcriptomic proteome in comparison with the complete $D$. coronatus proteome. Associated functional descriptions and test statistics are given in tabular format in Additional file 4.

Additional file 4. 1-8 cell expression-based proteome in comparison to the full D. coronatus proteome. Machine (including MS Excel) readable text file in tsv format with Blast2GO-derived statistical test results for enrichment of gene functions.

Additional file 5: Fig. S3. Binning of different combinations of replicates. Combining all four replicates the numbers of expressed sequences appear to saturate at about 6500 transcripts (see Table 2).

Additional file 6: Fig. S4. Phylogenetic tree representing GoLoco (Pfam ID PF02188) domain proteins of D. coronatus (D. C.), C. elegans (C. e.), human (H. s.), rat (R.n.) and mouse (M. m.).

\footnotetext{
Author's contributions

CK performed all major experiments, analyzed data, planned the study and wrote the manuscript. PHS performed experiments, analyzed data, planned the study and wrote and revised the manuscript. HK performed experiments and revised manuscript. HH performed experiments and revised manuscript. TV performed experiments. MK planned the study, analyzed data and revised manuscript. YK planned the study and revised manuscript. ES performed experiments, analyzed data, planned the study and wrote and revised the manuscript. All authors read and approved the final manuscript.
} 


\begin{abstract}
Author details
${ }^{1}$ Zoologisches Institut, Universität zu Köln, Cologne, NRW, Germany. ${ }^{2}$ Genetics, Evolution and Environment, University College London, London WC16BT, UK. ${ }^{3}$ National Institute of Genetics, Mishima, Japan. ${ }^{4}$ Present Address: Institute for Genetics, Universität zu Köln, Cologne, NRW, Germany. ${ }^{5}$ Present Address: Molecular Cell Biology, Institute I for Anatomy University Clinic Cologne, University of Cologne, Cologne, Germany.
\end{abstract}

\section{Acknowledgements}

We are grateful for the opportunity to use the Cheops computing cluster at the University of Cologne.

\section{Competing interests}

The authors declare that they have no competing interests.

\section{Availability of data and materials}

The D. coronatus genome and annotation are available through ensembl. caenorhabditis.org. Additional RNA-Seq data are available on SRA under the accession number PRJNA407968.

\section{Consent for publication}

All authors declare their consent with publication of the submitted manuscript.

\section{Ethics approval and consent to participate} Not applicable.

\section{Funding}

PHS was funded by the VolkswagenStiftung in their initiative for evolutionary biology and is currently supported by the European Research Council (ERC2012-AdG 322790) in a grant to Max Telford. CK was partly funded through the University of Cologne "Strings and Structures" research grant to Thomas Wiehe and Jürgen Rolshoven.

\section{Publisher's Note}

Springer Nature remains neutral with regard to jurisdictional claims in published maps and institutional affiliations.

\section{Received: 11 August 2017 Accepted: 10 October 2017} Published online: 18 October 2017

\section{References}

1. LahI V, Sadler B, Schierenberg E. Egg development in parthenogenetic nematodes: variations in meiosis and axis formation. Int J Dev Biol. 2006;50:393-7.

2. Hiraki H, Kagoshima H, Kraus C, Schiffer PH, Ueta Y, Kroiher M, et al. Genome analysis of Diploscapter coronatus: insights into molecular peculiarities of a nematode with parthenogenetic reproduction. BMC Genomics. 2017;18:478.

3. Schierenberg E, Sommer R. Reproduction and development in nematodes. In: Schmidt-Rhaesa A, editor. Handbook of zoology. Berlin: De Gruyter; 2014. p. 61-108.

4. True JR, Haag ES. Developmental system drift and flexibility in evolutionary trajectories. Evol Dev. 2001;3:109-19.

5. Schiffer PH, Kroiher M, Kraus C, Koutsovoulos GD, Kumar S, Camps الر et al. The genome of Romanomermis culicivorax: revealing fundamental changes in the core developmental genetic toolkit in Nematoda. BMC Genomics. 2013;14:923.

6. Schiffer PH, Nsah NA, Grotehusmann H, Kroiher M, Loer C, Schierenberg E. Developmental variations among panagrolaimid nematodes indicate developmental system drift within a small taxonomic unit. Dev Genes Evol. 2014;224:183-8.

7. Kiontke K, Fitch D. The phylogenetic relationships of Caenorhabditis and other rhabditids. In: The C. elegans Research Community, editor. WormBook; 2005. www.wormbook.org.

8. LahI V, Schulze J, Schierenberg E. Differences in embryonic pattern formation between Caenorhabditis elegans and its close parthenogenetic relative Diploscapter coronatus. Int J Dev Biol. 2009:53:507-15.
9. McCarter J, Bartlett B, Dang T, Schedl T. On the control of oocyte meiotic maturation and ovulation in Caenorhabditis elegans. Dev Biol. 1999;205:111-28.

10. Miller MA, Ruest PJ, Kosinski M, Hanks SK, Greenstein D. An Eph receptor sperm-sensing control mechanism for oocyte meiotic maturation in Caenorhabditis elegans. Genes Dev. 2003;17:187-200.

11. Miller M, Nguyen V, Lee M, Kosinski M, Schedl T, Caprioli R, et al. A sperm cytoskeletal protein that signals oocyte meiotic maturation and ovulation. Science. 2001:291:2144-7.

12. Heger $P$, Kroiher M, Ndifon N, Schierenberg E. Conservation of MAP kinase activity and MSP genes in parthenogenetic nematodes. BMC Dev Biol. 2010;10:51.

13. Hashimshony T, Wagner F, Sher N, Yanai I. CEL-Seq: single-cell RNA-Seq by multiplexed linear amplification. Cell Rep. 2012;2:1-8.

14. Wang J, Mitreva M, Berriman M, Thorne A, Magrini V, Koutsovoulos G, et al. Silencing of germline-expressed genes by DNA elimination in somatic cells. Dev Cell. 2012;23:1-9.

15. Simon J, Delmotte F, Rispe C, Crease T. Phylogenetic relationships between parthenogens and their sexual relatives: the possible routes to parthenogenesis in animals. Biol J Linn Soc. 2003;79:151-63.

16. Kiontke K, Gavin N, Raynes Y, Roehrig C, Piano F, Fitch D. Caenorhabditis phylogeny predicts convergence of hermaphroditism and extensive intron loss. Proc Natl Acad Sci. 2004;101:9003-8.

17. Kiontke KC, Félix M-A, Ailion M, Rockman MV, Braendle C, Penigault J-B, et al. A phylogeny and molecular barcodes for Caenorhabditis, with numerous new species from rotting fruits. BMC Evol Biol. 2011;11:339.

18. Neaves WB, Baumann P. Unisexual reproduction among vertebrates. Trends Genet. 2011;27:81-8.

19. Brenner S. The genetics of Caenorhabditis elegans. Genetics. 1974;77:71-94.

20. Schnabel $\mathrm{R}$, Hutter $\mathrm{H}$, Moerman D, Schnabel H. Assessing normal embryogenesis in Caenorhabditis elegans using a 4D microscope: variability of development and regional specification. Dev Biol. 1997;184:234-65.

21. Williams BD, Schrank B, Huynh C, Shownkeen R, Waterston RH. A genetic mapping system in Caenorhabditis elegans based on polymorphic sequence-tagged sites. Genetics. 1992;131:609-24.

22. Holterman M, Holovachov $\mathrm{O}$, van den Elsen $\mathrm{S}$, van Megen $\mathrm{H}$, Bongers T, Bakker J, et al. Small subunit ribosomal DNA-based phylogeny of basal Chromadoria (Nematoda) suggests that transitions from marine to terrestrial habitats (and vice versa) require relatively simple adaptations. Mol Phylogenet Evol. 2008;48:758-63.

23. Floyd R, Abebe E, Papert A, Blaxter M. Molecular barcodes for soil nematode identification. Mol Ecol. 2002:11:839-50.

24. Sonnenberg R, Nolte A, Tautz D. An evaluation of LSU rDNA D1-D2 sequences for their use in species identification. Front Zool. 2007:4:6.

25. Vrain TC. Restriction fragment length polymorphism separates species of the Xiphinema americanum Group. J Nematol. 1993;25:361-4.

26. Ronquist F, Huelsenbeck JP. MrBayes 3: bayesian phylogenetic inference under mixed models. Bioinformatics 2003;19(12):1572-4. doi:10.1093/ bioinformatics/btg180

27. Stamatakis A. RAxML-VI-HPC: maximum likelihood-based phylogenetic analyses with thousands of taxa and mixed models. Bioinformatics. 2006;22:2688-90.

28. Li L, Stoeckert CJ, Roos DS. OrthoMCL: identification of ortholog groups for eukaryotic genomes. Genome Res. 2003;13:2178-89.

29. C. elegans Sequencing Consortium. Genome sequence of the nematode C. elegans: a platform for investigating biology. Science. 1998;282:2012-8

30. Stein LD, Bao Z, Blasiar D, Blumenthal T, Brent MR, Chen N, et al. The genome sequence of Caenorhabditis briggsae: a platform for comparative genomics. PLoS Biol. 2003:1:E45.

31. Mortazavi A, Schwarz EM, Williams B, Schaeffer L, Antoshechkin I, Wold BJ, et al. Scaffolding a Caenorhabditis nematode genome with RNA-seq. Genome Res. 2010;20:1740-7.

32. Dieterich C, Clifton SW, Schuster LN, Chinwalla A, Delehaunty K Dinkelacker I, et al. The Pristionchus pacificus genome provides a unique perspective on nematode lifestyle and parasitism. Nat Genet. 2008:40:1193-8.

33. Srinivasan J, Dillman AR, Macchietto MG, Heikkinen L, Lakso M, Fracchia $\mathrm{KM}$, et al. The draft genome and transcriptome of Panagrellus redivivus 
are shaped by the harsh demands of a free-living lifestyle. Genetics. 2013;193:1279-95.

34. Jex AR, Liu S, Li B, Young ND, Hall RS, Li Y, et al. Ascaris suum draft genome. Nature. 2011;479:1-8.

35. Blüthgen N, Brand K, Cajavec B, Swat M, Herzel H, Beule D. Biological profiling of gene groups utilizing Gene Ontology. Genome Inform. 2005;16:106-15.

36. Conesa A, Götz S, García-Gómez JM, Terol J, Talón M, Robles M. Blast2GO: a universal tool for annotation, visualization and analysis in functional genomics research. Bioinformatics. 2005;21:3674-6.

37. Holterman $M$, van der Wurff $A$, van den Elsen $S$, van Megen $H$, Bongers T, Holovachov O, et al. Phylum-wide analysis of SSU rDNA reveals deep phylogenetic relationships among nematodes and accelerated evolution toward crown clades. Mol Biol Evol. 2006;23:1792-800.

38. De Ley P, Blaxter ML. Systematic position and phylogeny. In: Lee DL, editor. The biology of nematodes. London: Taylor and Francis; 2002. p. $1-30$.

39. Sievers F, Wilm A, Dineen D, Gibson TJ, Karplus K, Li W, et al. Fast, scalable generation of high-quality protein multiple sequence alignments using Clustal Omega. Mol Syst Biol. 2011;7:1-6.

40. Finn RD, Coggill P, Eberhardt RY, Eddy SR, Mistry J, Mitchell AL, et al. The Pfam protein families database: towards a more sustainable future. Nucleic Acids Res. 2016;44:D279-85.

41. Darriba D, Taboada GL, Doallo R, Posada D. ProtTest 3: fast selection of best-fit models of protein evolution. Bioinformatics. 2011;27:1164-5.

42. Baugh LR, Hill AA, Brown EL, Hunter CP. Quantitative analysis of mRNA amplification by in vitro transcription. Nucleic Acids Res. 2001;29:E29.

43. Bolger AM, Lohse M, Usadel B. Trimmomatic: a flexible trimmer for Illumina sequence data. Bioinformatics. 2014;30:2114-20.

44. Haas BJ, Papanicolaou A, Yassour M, Grabherr M, Blood PD, Bowden J, et al. De novo transcript sequence reconstruction from RNA-Seq: reference generation and analysis with Trinity. Nat Protoc. 2013;8:1494-512.

45. Langmead B, Salzberg SL. Fast gapped-read alignment with Bowtie 2. Nat Methods. 2012;9:357-9.

46. Wang J, Garrey J, Davis RE. Transcription in pronuclei and one- to four-cell embryos drives early development in a nematode. Curr Biol. 2014:24:124-33.

47. LiW, Godzik A. Cd-hit: a fast program for clustering and comparing large sets of protein or nucleotide sequences. Bioinformatics. 2006:22:1658-9.

48. Kent W. BLAT-the BLAST-like alignment tool. Genome Res. 2002;12:656.

49. Altschul SF, Gish W, Miller W, Myers EW. Basic local alignment search tool. J Mol Biol. 1990;215:403-10.

50. Mitreva M, Jasmer DP, Zarlenga DS, Wang Z, Abubucker S, Martin J, et al. The draft genome of the parasitic nematode Trichinella spiralis. Nat Genet. 2011:43:228-35.

51. Thompson JD, Higgins DG, Gibson TJ. CLUSTAL W: improving the sensitivity of progressive multiple sequence alignment through sequence weighting, position-specific gap penalties and weight matrix choice. Nucleic Acids Res. 1994;22:4673-80.

52. Quevillon E, Silventoinen V, Pillai S, Harte N, Mulder N, Apweiler R, et al. InterProScan: protein domains identifier. Nucleic Acids Res. 2005;33:W116-20.

53. Zhang Z, Li J, Zhao X-Q, Wang J, Wong GK-S, Yu J. KaKs_Calculator: calculating $\mathrm{Ka}$ and $\mathrm{Ks}$ through model selection and model averaging. Genomics Proteomics Bioinformatics. 2006:4:259-63.

54. Li H, Handsaker B, Wysoker A, Fennell T, Ruan J, Homer N, et al. The sequence alignment/map format and SAMtools. Bioinformatics. 2009;25:2078-9.

55. Danecek P, Auton A, Abecasis G, Albers CA, Banks E, DePristo MA, et al. The variant call format and VCFtools. Bioinformatics. 2011;27:2156-8.

56. Greenstein D. Control of oocyte meiotic maturation and fertilization. In: The C. elegans Research Community, editor. WormBook; 2005. www. wormbook.org.

57. Kimble J, Crittenden S. Germline proliferation and its control. In: The C. elegans Research Community, editor. WormBook; 2005. www.wormbook.org.

58. Hechler HC. Postembryonic development and reproduction in Diploscapter coronatus (Nematoda, Rhabditidae). Washington: Helminth. Society; 1968. p. 24-30
59. Albertson D, Rose AM, Villeneuve AM. Chromosome organization, mitosis, meiosis. In: Riddle DL, Blumenthal T, Meyer BJ, Priess JR, editors, The nematode C. elegans, II. Cold Spring Harbor: Cold Spring Harbor Laboratory Press; 1997. p. 47-78.

60. Gönczy P, Rose LS. Asymmetric cell division and axis formation in the embryo. In: The C. elegans Research Community, editor. WormBook; 2005. www.wormbook.org.

61. Cowan CR, Hyman AA. Centrosomes direct cell polarity independently of microtubule assembly in C. elegans embryos. Nature. 2004;431:92-6.

62. Tsou M-FB, Hayashi A, DeBella LR, McGrath G, Rose LS. LET-99 determines spindle position and is asymmetrically enriched in response to PAR polarity cues in C. elegans embryos. Development. 2002;129:4469-81.

63. Rose L, Gönczy P. Polarity establishment, asymmetric division and segregation of fate determinants in early C. elegans embryos. In: The $C$. elegans Research Community, editor. WormBook; 2014. www.wormbook.org.

64. Srinivasan DG, Fisk RM, Xu H, van den Heuvel S. A complex of LIN-5 and GPR proteins regulates $G$ protein signaling and spindle function in $C$. elegans. Gene Dev. 2003;17:1225-39.

65. Nguyen-Ngoc T, Afshar K, Gönczy P. Coupling of cortical dynein and G [alpha] proteins mediates spindle positioning in Caenorhabditis elegans. Nat Cell Biol. 2007;9:1294-302.

66. Schierenberg E. Reversal of cellular polarity and early cell-cell interaction in the embryo of Caenorhabditis elegans. Dev Biol. 1987:122:452-63.

67. Cheng NN, Kirby CM, Kemphues KJ. Control of cleavage spindle orientation in Caenorhabditis elegans: the role of the genes par-2 and par-3. Genetics. 1995;139:549-59.

68. Rose LS, Kemphues K. The let-99 gene is required for proper spindle orientation during cleavage of the C. elegans embryo. Development. 1998;125:1337-46.

69. Altaf M, Auger A, Monnet-Saksouk J, Brodeur J, Piquet S, Cramet M, et al. NuA4-dependent acetylation of nucleosomal histones $\mathrm{H} 4$ and $\mathrm{H} 2 \mathrm{~A}$ directly stimulates incorporation of H2A.Z by the SWR1 complex. J Biol Chem. 2010;285:15966-77.

70. Whittle CM, McClinic KN, Ercan S, Zhang X, Green RD, Kelly WG, et al. The genomic distribution and function of histone variant HTZ-1 during C. elegans embryogenesis. PLoS Genet. 2008;4:e1000187.

71. Welch D, Meselson M. Evidence for the evolution of bdelloid rotifers without sexual reproduction or genetic exchange. Science. 2000;288:1211-5.

72. Butlin R. Evolution of sex: the costs and benefits of sex: new insights from old asexual lineages. Nat Rev Genet. 2002;3:311-7.

73. Blanc-Mathieu R, Perfus-Barbeoch L, Aury J-M, Da Rocha M, Gouzy J, Sallet $\mathrm{E}$, et al. Hybridization and polyploidy enable genomic plasticity without sex in the most devastating plant-parasitic nematodes. PLoS Genet. 2017:13:e1006777-36.

74. Kimble J, Crittenden SL. Controls of germline stem cells, entry into meiosis, and the sperm/oocyte decision in Caenorhabditis elegans. Ann Rev. 2007;23:405-33.

75. Kim S, Spike C, Greenstein D. Control of oocyte growth and meiotic maturation in Caenorhabditis elegans. Germ cell development in C. elegans. Adv Exp Med Biol. 2013;757:277-320.

76. Lahl V, Halama C, Schierenberg E. Comparative and experimental embryogenesis of Plectidae (Nematoda). Dev Genes Evol. 2003;213:18-27.

77. Brown HW, Cort WW. The egg production of Ascaris lumbricoides. J Parasitol. 1927;14:88-90.

78. Viera A, Page J, Rufas JS. Inverted meiosis: the true bugs as a model to study meiosis. Genome Dyn. 2009;5:137-56.

79. Beatty A, Morton D, Kemphues K. The C. elegans homolog of Drosophila Lethal giant larvae functions redundantly with PAR-2 to maintain polarity in the early embryo. Development. 2010;137:3995-4004.

80. Wirtz-Peitz F, Knoblich JA. Lethal giant larvae take on a life of their own. Trends Cell Biol. 2006:16:234-41.

81. Knoblich JA. Mechanisms of asymmetric stem cell division. Cell. 2008;132:583-97.

82. Roegiers F, Jan YN. Asymmetric cell division. Curr Opin Cell Biol. 2004;16:195-205. 
83. Hyman AA. Centrosome movement in the early divisions of Caenorhabditis elegans: a cortical site determining centrosome position. J Cell Biol. 1989;109:1185-93.

84. Hyman AA, White JG. Determination of cell division axes in the early embryogenesis of Caenorhabditis elegans. J Cell Biol. 1987;105:2123-35.

85. Schulze J, Schierenberg E. Cellular pattern formation, establishment of polarity and segregation of colored cytoplasm in embryos of the nematode Romanomermis culicivorax. Dev Biol. 2008;315:426-36.

86. Brauchle M, Kiontke K, MacMenamin P, Fitch DHA, Piano F. Evolution of early embryogenesis in rhabditid nematodes. Dev Biol. 2009;335:253-62

87. Tipney H, Hunter L. An introduction to effective use of enrichment analysis software. Hum Genomics. 2010;4:202-6.

88. Huang DW, Sherman BT, Lempicki RA. Bioinformatics enrichment tools: paths toward the comprehensive functional analysis of large gene lists. Nucleic Acids Res. 2009:37:1-13.

89. Lynch M. Destabilizing hybridization, general-purpose genotypes and geographic parthenogenesis. Quart Rev Biol. 1984;59:257-90.

90. Lunt DH. Genetic tests of ancient asexuality in root knot nematodes reveal recent hybrid origins. BMC Evol Biol. 2008:8:194-216.

91. Zhou T, Gu W, Wilke CO. Detecting positive and purifying selection at synonymous sites in yeast and worm. Mol Biol Evol. 2010;27:1912-22.

92. Lunt DH, Kumar S, Koutsovoulos G, Blaxter ML. The complex hybrid origins of the root knot nematodes revealed through comparative genomics. Peer J. 2014;2:e356.

93. Szitenberg A, Salazar-Jaramillo L, Blok VC. Comparative genomics of apomictic root-knot nematodes: hybridization, ploidy, and dynamic genome change. bioRxiv 2017.

94. Schiffer PH, Danchin E, Burnell AM, Schiffer A-M, Creevey C, Wong S, et al. Signatures of the evolution of parthenogenesis and cryptobiosis in the genomes of panagrolaimid nematodes. bioRxiv 2017.
95. Nicholas WL. The biology of free-living nematodes. Oxford: Oxford University Press; 1984

96. Nigon V. Développement et reproduction des nématodes. In: Grasse PP, editor. Traité de Zoology IV. Paris: Masson et Cie; 1965. p. 218-94.

97. Fradin H, Kiontke K, Zegar C, Gutwein M, Lucas J, Kovtun M, et al. Genome architecture and evolution of a unichromosomal asexual nematode. Curr Biol. 2017 (Epub ahead of print).

98. Schubert I. Chromosome evolution. Curr Opin Plant Biol. 2007;10:109-15.

99. Wienberg J. The evolution of eutherian chromosomes. Curr Opin Genet Dev. 2004;14:657-66.

100. van Beneden E. Recherches sur la maturation de l'œuf et la fécondation et la division cellulaire. Arch Biol. 1883;4:265-640.

101. Félix M-A, Braendle C, Cutter AD. A streamlined system for species diagnosis in Caenorhabditis (Nematoda: Rhabditidae) with name designations for 15 distinct biological species. PLoS ONE. 2014;9:e94723.

102. Schierenberg E, Schulze J. Many roads lead to Rome: different ways to construct a nematode. In: Minelli A, Fusco G, editors. Key themes in evolutionary developmental biology. Cambridge: Cambridge University Press; 2008. p. 261-80

103. Schulze J, Schierenberg E. Evolution of embryonic development in nematodes. EvoDevo. 2011:2:18

104. Tintori SC, Osborne Nishimura E, Golden P, Lieb JD, Goldstein B. A transcriptional lineage of the early C. elegans embryo. Dev Cell. 2016;38:430-44.

\section{Submit your next manuscript to BioMed Central and we will help you at every step:}

- We accept pre-submission inquiries

- Our selector tool helps you to find the most relevant journal

- We provide round the clock customer support

- Convenient online submission

- Thorough peer review

- Inclusion in PubMed and all major indexing services

- Maximum visibility for your research

Submit your manuscript at www.biomedcentral.com/submit

O Biomed Central 